\title{
Reaction pathway of tryptophanase-catalyzed L-tryptophan synthesis from D-serine
}

Akihiko Shimada $^{\mathrm{a}, *}$, Haruka Ozaki ${ }^{\mathrm{a}}$, Takeshi Saito ${ }^{\mathrm{b}}$ and Noriko Fujii ${ }^{\mathrm{b}}$

${ }^{a}$ Sustainable Environmental Studies, Graduate School of Life and Environmental

Sciences, University of Tsukuba, Tsukuba, Ibaraki 305-8572, Japan

${ }^{\mathrm{b}}$ Research Reactor Institute, Kyoto University, Kumatori, Osaka 590-0494, Japan

*Corresponding author. Tel.: +81 2988534367.

E-mail address: ashimada@envr.tsukuba. ac.jp

\begin{abstract}
Tryptophanase, L-tryptophan indole-lyase with extremely absolute stereospecificity, can change the stereospecificity in concentrated diammonium hydrogenphosphate solution. While tryptophanase is inert to $\mathrm{D}$-serine in the absence of diammonium hydrogenphosphate, it can undergo L-tryptophan synthesis from D-serine along with indole in the presence of it. It has been well known that tryptophanase synthesizes L-tryptophan from L-serine through a $\beta$-substitution mechanism of the ping-pong type. However, a metabolic pathway of L-tryptophan synthesis from D-serine has remained unclear. The present study aims to elucidate it. Diammonium hydrogenphosphate plays a role in the emergence of catalytic activity on D-serine. The salt gives tryptophanase a
\end{abstract}


small conformational change, which makes it possible to catalyze D-serine. Tryptophanase-bound D-serine produces L-tryptophan synthesis by $\beta$-replacement reaction via the enzyme-bound aminoacrylate intermediate. Our result will be valuable in studying the origin of homochirality.

Keywords: Stereospecificity, Diammonium hydrogenphosphate, Tryptophanase, D-serine, L-tryptophan synthesis, Origin of homochirality

\section{Introduction}

Life makes exclusive selection of L-amino acids from DL-amino acids to establish the homochiral biological world. It is enzyme stereospecificity that undertakes this very difficult task. If the enzyme stereospecificity is ambiguous or unstable, the right type of two antipodes cannot be accurately selected. When a polypeptide is randomly-mixed with L- and D- amino acid, it is impossible to build up a protein with regularly-folded stereostructure because the polypeptide chain cannot be elongated in an enantiometrically racemic mixture [1]. The enzyme stereospecificity must be both stable and absolute. This absoluteness is important in biosynthesis rather than in biodegradation. A stable supply of homochiral molecules is especially essential in synthesizing biological substances with a highly-organized stereostructure such as protein or polysaccharide. In other words, life cannot survive without the 
stereospecificity [2]. Thereby, we have a prevailing orthodoxy that the invariance principle of it goes on for eternity. However, there is no theoretical evidence to support the principle. Few related discussions have been presented about the characterization of the stereospecificity. The major theory is three-point theory [3], but it is difficult to understand the whole picture of complex asymmetric interactions between substrate and many amino acid residues participating at the enzyme active site on the basis of this theory [4]. Unfortunately, an understanding of the stereospecificity has still remained ambiguous. Further study will be required to define chiral recognition precisely.

We have so far studied the stereospecificity of tryptophanase (TPase), with the aim of acquiring a better understanding of the stereospecificity. TPase is an enzyme with extremely tight stereospecificity, cleaving L-tryptophan into indole, pyruvate and ammonia. Despite broad substrate specificity to many other $\beta$-substituted L-amino acids and their analogs [5], TPase exhibits no activity on D-tryptophan. However, it becomes active to D-tryptophan degradation in highly concentrated diammonium hydrogen phosphate (DAP) solution, which fact indicates that TPase has flexible stereospecificity. The details of this reaction are presented elsewhere [6-9]. In the studies focused on the origins of life, synthetic reactions have greater significance than degradative reaction because life is thought to be born as the result of accumulation of countless homochiral synthetic reactions. It is logical to think that enzyme stereospecificity should be more precise in synthesis than degradation in chiral selection of biomolecules for survival. In this context, it seems to become more difficult to find the flexible stereospecificity in synthesis reactions. Nonetheless, we attempt to seek out because it is expected to be invaluable in the study of the origin of homochirality. So, we have our eyes on TPase-bound $\alpha$-aminoacylate as an intermediate common to both D-tryptophan 
degradation and tryptophan synthesis from L-serine [10]. TPase can synthesize L-tryptophan from L-serine and indole via the intermediate by $\beta$-substitution reaction with ping-pong type mechanism [11]. TPase also has absolute stereoselectivity in this synthesis, and thereby no activity on D-serine. However, DAP induces stereoselective conversion to make D-tryptophan active, and then D-tryptophan is degraded to indole via the intermediate [12]. It is rational to deduce that TPase receives the same change from DAP in tryptophan synthesis. If D-serine binds with TPase to form TPase-bounded $\alpha$-aminoacylate in the presence of DAP, it seems quite likely that tryptophan will be synthesized from D-serine with added indole. In fact, we previously reported L-tryptophan was synthesized from D-serine [13]. However, this reaction pathway has remained undetermined. There are three possible pathways in the reaction. The first possible pathway is L-tryptophan synthesis through chemical D-serine racemization. This means that not D-serine but L-serine participates in the synthetic reaction. The second one is a $\beta$-elimination reaction through which the $\mathrm{C}^{\beta}-\mathrm{OH}$ of $\mathrm{D}$-serine is eliminated with deamination to form pyruvate, which is recombined with indole and ammonia to produce L-tryptophan. $\beta$-replacement reaction by which the $\mathrm{C}^{\beta}-\mathrm{OH}$ is substituted to indole is lastly proposed. This paper aims to determine which reaction pathway synthesizes L-tryptophan from D-serine in the presence of DAP.

\section{Materials and methods}

\subsection{Materials and reagents}


E. coli apotryptophanase purchased from Sigma-Aldrich Co. (St. Louis, MO, USA), was easily soluble in aqueous solution. SDS-PAGE showed a single band at MW 52500, indicating that it was completely pure. $1 \mathrm{mg}$ of apotryptophanase released 75 to $150 \mu \mathrm{g}$ of indole from L-tryptophan in 10 min with $\mathrm{pH} 8.3$ at $37^{\circ} \mathrm{C}$ in the presence of $0.04 \mathrm{mM}$ of pyridoxal 5'-phosphate. Monoammonium dihydrogen phosphate (MAP), DAP, and indole were purchased from Wako Pure Chemical Industries (Osaka, Japan), L-serine and D-serine from Peptide Institute Inc. (Osaka, Japan), and pyridoxal 5'-phosphate from Nakalai Tesque Inc. (Kyoto, Japan). Unless otherwise noted, all chemicals were high reagent grade, purchased from Wako Pure Chemical Industries. All glassware was washed by soaking for more than three days in a detergent, Clean 99 CL, and were thoroughly rinsed and then dried in an oven. All aqueous solutions were prepared from deionized and ceramics-distilled water.

\subsection{Tryptophan production from $D$ - and L-serine}

$100 \mathrm{mM}$ potassium phosphate buffer of $\mathrm{pH} 8.3$ (PB) was always used as standard buffer solution. 6.2 M DAP solution was diluted with $\mathrm{PB}$ to obtain the required concentrations. For example, 1.2 M was prepared by adding 4.2 volumes of $\mathrm{PB}$ to 1 volume of the 6.2 M DAP solution. Reaction mixtures included $0.4 \mathrm{mM}$ pyridoxal 5'-phosphate, $20 \mathrm{mM}$ L-serine or D-serine, $5.4 \mathrm{mM}$ indole, and $0.46 \mu \mathrm{M}$ TPase with 0 to 4.3 $\mathrm{M}$ concentration of DAP in the reaction temperature range of 30 to $80{ }^{\circ} \mathrm{C}$. Reactions were performed for $6 \mathrm{hr}$ at the required DAP concentration and temperature in a Dry Thermo Unit DTU-1B (Taitec, Tokyo, Japan). The total volume of the reaction mixture was $2 \mathrm{~mL}$ per tube. The reaction was stopped by fast cooling to $0{ }^{\circ} \mathrm{C}$ on ice, and then the product in the reaction mixture was analyzed. Unless otherwise noted, the same 
concentration of these reagents and reaction time was always applied to other experiments.

Reaction product was determined resolving with cellulose thin layer chromatography (TLC Cellulose, plastic sheets $20 \times 20 \mathrm{~cm}$, Merck KgaA, Dannstadt, Germany) and Crownpack CR (+) Resolution HPLC column (Daicel Industry Ltd., Tokyo, Japan). All proteinic amino acids except glycine can be resolved on cellulose thin layer chromatography at lower temperature using development solvents such as $n$-butanol/pyridine/water or ethanol/pyridine/water [14-16]. In the cellulose thin layer chromatography, the composition of developing solvent was $n$-butanol : pyridine : water $=1: 1: 1$. All cellulose thin layer plates were triply predeveloped by washing with this same solvent to remove contaminants that disturbed development and ninhydrin chromogenic reaction. Then the plates were dried in the oven at $50{ }^{\circ} \mathrm{C}$ for $1 \mathrm{hr}$, stored in a plastic desiccator with silica gel until use. Reaction was stopped by adding the same volume of n-butanol as reaction mixture, vigorously mixed, then immediately centrifuged at about $1,000 \mathrm{G}$ for $10 \mathrm{~min}$. Centrifuging caused 3 layers to form, - a butanol layer (supernatant), a denatured enzyme layer (middle), and an aqueous layer (bottom). $5 \mu \mathrm{L}$ of the supernatant was spotted onto the plates, which were developed at a constant temperature of $5{ }^{\circ} \mathrm{C}$ in the refrigerator. After development, the plates were dried up, and immediately sprayed by ninhydrin reagent for colorimetry. The $\mathrm{R}_{\mathrm{f}}$ value of purple spot, specific to amino acid, was compared to that of D- or L-tryptophan. Although cellulose thin layer chromatography analyses showed that the reaction product was an amino acid with $\mathrm{R}_{\mathrm{f}}$ equal to L-tryptophan, HPLC analysis by use of resolution column was still necessary to confirm it exactly. The product was thus resolved on a resolution column, Crownpack CR (+) Resolution HPLC column (Daicel Industry Ltd., 
Tokyo, Japan) to be analysed with UV and CD monitor (CD-1595 detector, Jasco, Tokyo, Japan). Perchloric acid was added dropwise to a few liters of distilled water with vigorous stirring at room temperature until $\mathrm{pH}$ decreased to 2.0 , and used as an eluting buffer for HPLC. Reactions were performed in optimal conditions. When L-serine was substrate, reaction temperature was $50{ }^{\circ} \mathrm{C}$ in the absence of DAP. When D-serine was substrate, it was $60{ }^{\circ} \mathrm{C}$ in the presence of $1.2 \mathrm{M}$ DAP. $20 \mu \ell$ of the reaction mixture cooled at $0{ }^{\circ} \mathrm{C}$ on ice was filtered through Ultrafree-MC 5000 NMWL Filter Unit (Millipore Co. Bedford, MA, USA) by centrifugation, immediately injected into the resolution column at room temperature, and resolved at a flow rate of $0.6 \mathrm{~mL} / \mathrm{min}$ with the eluting buffer running with an HPLC pump (635 Liquid Chromatogram, Hitachi, Ltd., Tokyo, Japan) through a Degasser (DG-980-50, Jasco, Tokyo, Japan). The eluant was monitored with an UV and CD monitor at a wavelength of $\lambda=239 \mathrm{~nm}$. Circular dichroism $(\mathrm{CD})$ is the most effective method for determining the enantiomeric form of tryptophan. Ellipticity of the eluting buffer was set up to zero at a wavelength of $\lambda=239$ $\mathrm{nm}$, so that the ellipticity of D- and L-tryptophan had a negative and positive peak, respectively. Vertical axis on chromatograms was represented in absorbance and millidegree of ellipticity [ $\theta]$ at $\lambda=239 \mathrm{~nm}$. TPase activity on tryptophan synthesis from L- or D-serine was calculated from a peak area of the reactant on UV chromatogram on the basis of a peak area of $4.9 \mathrm{mM}$ L-tryptophan. Indole, DAP and pyridoxal 5'-phosphate were eluted as a sharp large peak near void volume.

\subsection{Fluorescence and circular dichroism photometer}

Bis-ANS (4,4'-dianilino-1,1'-binaphthalene-5,5'-disulfonic acid, $\varepsilon=23 \times 10^{3} \mathrm{~cm}^{-1} \mathrm{M}^{-1}$ at $395 \mathrm{~nm})$ is a hydrophobic fluorescent probe indicating the existence of 
solvent-exposed hydrophobic domains in a protein's structure. A stock solution of bis-ANS was prepared to a final concentration of $0.5 \mu \mathrm{M}$ by dissolving $0.3 \mathrm{mg}$ in $1 \mathrm{~mL}$ of PB before experiments. $20 \mu \mathrm{L}$ of bis-ANS stock was added to a $3 \mathrm{~mL}$ solution of $\mathrm{PB}$, MAP and DAP solutions of 1.2 M concentration including $0.46 \mu \mathrm{M}$ apoTPase and 1.1 $\mathrm{mM}$ pyridoxal 5'-phosphate. These aliquots were left to stand at room temperature for at least $15 \mathrm{~min}$ before measurement, and then placed in a cuvette in a fluorescence spectrophotometer (Hitachi F4500, Tokyo, Japan). Emission from 420 to 650nm was measured at an excitation wavelength of $395 \mathrm{~nm}$ [17]. The fluorescence of bis-ANSbound TPase was calculated by subtracting a blank prepared identically but without TPase. Relative fluorescence intensity was measured in arbitrary units.

In a circular dichroism spectrophotometer (J805, Jasco, Tokyo, Japan), a $300 \mu \mathrm{L}$ solution including $\mathrm{PB}, 1.2 \mathrm{M}$ MAP or DAP with $0.46 \mu \mathrm{M}$ apoTPase and $1.1 \mathrm{mM}$ pyridoxal 5'-phosphate was injected in a $0.1 \mathrm{~cm}$ path length cell. Spectra were recorded at wavelengths from 200 to $400 \mathrm{~nm}$ at room temperature. Five scans were accumulated per spectrum, averaged, and expressed as molar ellipticity in degrees $\mathrm{cm}^{2} \mathrm{dmol}^{-1}$. The CD spectra were analyzed with a CDPro software package and calculated by subtracting a blank prepared identically but without TPase.

\subsection{Determination of enantiomeric form of $D$-serine}

Prior to experiment, perchloric acid was added dropwise to a few liters of distilled water with vigorous stirring at room temperature until $\mathrm{pH}$ decreased to 2.0 , and used as an eluent for HPLC. Reaction mixture including D-serine, 1.2 M DAP and pyridoxal $5^{\prime}$-phosphate was exposed to $60^{\circ} \mathrm{C}$ for $6 \mathrm{hr}$. The reaction mixture was filtered through Amicon Ultra Centrifugal Filters cutting more than MW 3000 (Millipore, Corrigtwohill, 
Co. Cork, Ireland), immediately injected on a Crownpack CR (+) Resolution HPLC column equilibrated with the degassed eluent through a Degasser, and eluted at a flow rate of $0.1 \mathrm{~mL} / \mathrm{min}$ at $0{ }^{\circ} \mathrm{C}$ with an HPLC pump. D-serine in the reaction mixture was resolved on the column. Ellipticity [ $\theta]$ was monitored at $\lambda=230 \mathrm{~nm}$ with $\mathrm{CD}$ detector, represented in units of mdeg. Ellipticity of the eluent was set up to zero at a wavelength of $\lambda=230 \mathrm{~nm}$, so the peaks of D-serine and L-serine appeared on the negative and positive side, respectively.

\subsection{Pyruvate assay}

Reaction mixtures including $0.4 \mathrm{mM}$ pyridoxal 5'-phosphate, $20 \mathrm{mM}$ D-serine, 0.46 $\mu \mathrm{M}$ TPase and 1.2 M DAP with 0 to $8 \mathrm{mM}$ indole were prepared to assay pyruvate production, placed in a Dry Thermo Unit DTU-1B at $60{ }^{\circ} \mathrm{C}$ for 0 to $8 \mathrm{hr}$. The total volume of the reaction mixture was $2 \mathrm{~mL}$ per tube. The reaction was stopped by fast cooling to $0{ }^{\circ} \mathrm{C}$ on ice, and the pyruvate produced was immediately analyzed. Pyruvate, a product of $\beta$-elimination reaction from $\mathrm{D}$-serine in the presence of DAP, was determined by an enzymatic assay. In the enzyme assay, pyruvate production was determined from the decrease in absorbance at $\lambda=340 \mathrm{~nm}$ in the presence of excess NADH and lactate dehydrogenase by use of F-kit Pyruvate (J. K. International, Tokyo, Japan). The range of pyruvate detection was limited from 0.2 to $5.7 \mathrm{mM}$.

\section{Results and discussion}

\subsection{Determination of L-tryptophan synthesized from $L$ - or D-serine}


As seen in the middle of Figure 1 , the $\mathrm{R}_{\mathrm{f}}$ values of $\mathrm{D}$ - or L-tryptophan were calculated to be 0.63 and 0.58 , respectively. DL-serine could not be resolved in the solvent used. D- or L-serine was emerged in all of lane $1-4$ as purple spots with $\mathrm{R}_{\mathrm{f}}=$ 0.34. A purple spot $\left(R_{f}=0.39\right)$ on the serine spot in lane 4 was unidentified. Two ninhydrin-colored purple spots, specific to amino acids, were seen in lane $1 . \mathrm{R}_{\mathrm{f}}$ of the upper and lower spots corresponded to L-tryptophan and L-serine, respectively. L-tryptophan was synthesized from L-serine and indole on TPase, as is well known. D-serine was provided in lanes 2 and 3, but they could not synthesize tryptophan at all because either TPase or DAP was removed from reaction mixture in their lanes Tryptophan-like purple spot appeared in lane 4 when TPase reacted with D-serine in the presence of DAP. $R_{f}$ value of the product was 0.58 , corresponding to that of L-tryptophan. This result showed DAP was necessary for TPase to synthesize tryptophan from D-serine.

4.9 mM D-and L-tryptophan were resolved in advance for reference in Fig. 2(a) and (b). The retention time of D- and L-tryptophan was 23 and $30 \mathrm{~min}$, respectively. D-tryptophan appeared on the negative side in ellipticity, but L-tryptophan on the positive side in Fig. 2(b). When TPase reacted with L-serine in Fig. 2(c), a peak with a retention time of $30 \mathrm{~min}$ appeared and its ellipticity was positive (Fig. 2(d)). This indicated the peak was L-tryptophan. While TPase could synthesize nothing from D-serine in the absence of DAP (Fig. 2(e)), it could synthesize a reaction product with positive ellipticity at a retention time of $30 \mathrm{~min}$, L-tryptophan, from D-serine in the presence of DAP (Fig. 2(f, g)). The present analytical methods were powerful enough to determine L-tryptophan synthesized from D-serine. 


\subsection{Optimal conditions of L-tryptophan synthesis from D-serine}

As the previous report [13] didn't compare enzyme activity on D-serine against that on L-serine, it was investigated in Figures 3 and 4. TPase-catalyzed L-tryptophan synthesis from L- or D-serine was analyzed at a fixed reaction temperature of $60{ }^{\circ} \mathrm{C}$ with DAP concentrations, or at a fixed DAP concentration of $1.2 \mathrm{M}$ with reaction temperature. Fig. 3 shows L-tryptophan synthesis from L- or D-serine dependent on DAP concentrations. L-tryptophan synthesis from L-serine was a maximum of $2.5 \mathrm{mM}$ in the absence of DAP, consistently declining with increasing DAP. When the substrate was D-serine, L-tryptophan synthesis increased with DAP concentrations. It reached a maximum value of $0.8 \mathrm{mM}$ at $1.2 \mathrm{M}$ concentration of DAP. L-tryptophan production from D-serine was $32 \%$ of that from L-serine. DAP exerted an inhibitory action on L-tryptophan synthesis from L-serine, but activated L-tryptophan synthesis from D-serine. Fig. 4 shows the influence of temperature on the reaction. L-tryptophan synthesis from L-serine was analyzed at 0 or $1.2 \mathrm{M}$ of DAP, but that from D-serine only at 1.2 M. L-tryptophan synthesis from L-serine increased with reaction temperature, reached its maximum value at $50{ }^{\circ} \mathrm{C}(0 \mathrm{M})$ and $55^{\circ} \mathrm{C}(1.2 \mathrm{M})$ and dropped at higher temperatures. L-tryptophan synthesis from D-serine also went through a similar process except an optimal reaction temperature of $60^{\circ} \mathrm{C}$. L-tryptophan synthesis from D-serine was optimal when a concentration of DAP and a reaction temperature was $1.2 \mathrm{M}$ and 60 ${ }^{\circ} \mathrm{C}$, respectively

\subsection{Spectrophotometerical analyses of conformational change}

Tryptophanase is originally an enzyme with absolute stereospecificity towards L-tryptophan or L-serine. However, Fig. 3 and 4 showed the activity on D-serine 
emerged in the presence of DAP. If we explain this cause on the basis of Popjak's three point theory [3], TPase conformation must be drastically upset to activate D-serine antipodal to L-serine. We investigated how the protein conformation changed by use of both fluorescence and circular dichroism spectrophotometers. The relative fluorescence intensity of bis-ANS-bound TPase was measured in PB, MAP or DAP solution of $1.2 \mathrm{M}$. Since there was the possibility that the small difference of fluorescent spectra might be produced by the difference of polarity of the solvent, we compared the fluorescence spectra of TPase-free DAP and PB solutions to check it prior to measurement. No fluorescence was emitted in either solution. Fluorescent spectrum didn't depend on the polarity. Fluorescence intensity was the highest in MAP, followed by DAP and PB (Fig. 5). The intensity had little difference between PB and DAP except that the maximum wavelength of DAP shifted to the left side by about $10 \mathrm{~nm}$ from that of PB. Unlike PB and DAP, a large difference was found in MAP. MAP irreversibly unfolds to deactivate TPase. The effect of the increasing exposure of hydrophobic amino acids is known to be closely related to aggregation of proteins, and any unfolding undoubtedly leads to the loss of all activity [18]. Since the unfolding of TPase was connected with the increasing exposure of hydrophobic amino acids, the fluorescent intensity increased drastically in response to it. Fluorescence spectral data showed that the conformational change of TPase was much larger in MAP than in DAP. The same result was also obtained from circular dichroism spectra of TPase in PB, DAP and MAP solutions. Spectral changes in the 200-250 $\mathrm{nm}$ region among three solutions arise from structural changes of $\alpha$-helix and $\beta$-sheet of TPase because their peaks are in the 205-225 nm region [19]. In Fig. 6, the CD spectrum of DAP was almost close or similar to that of PB in the $200-250 \mathrm{~nm}$ region, but the two CD spectra were not completely same. In fact, slight difference was 
seen in the wavelength range of $205-225 \mathrm{~nm}$. It reflects any subtle conformational change in secondary structure. A CD spectrum of TPase in MAP differed distinctively from those of PB and DAP. MAP caused irreversible full loss of the catalytic activity, which probably gave larger structural changes in secondary structure. We previously performed kinetic studies of D-tryptophan degradation reaction [9]. We reported there that TPase returned to the original activity, or TPase activity in PB when TPase was moved from DAP to PB solution, and the reversible activity meant the conformation reverted back to the first. Here the concentration of 1.2M DAP including TPase was diluted with PB by $12 \mathrm{mM}$ in which concentration the effect of DAP on TPase activity could be neglected, and then we examined the L-Trp synthesis activity of TPase. The activity, which centupled an actual activity value obtained in experiment, was compared with TPase activity in PB. The two activities were nearly identical. This is the same result as in the case of D-Trp degradation, in which the conformational change was reversible between DAP and PB. DAP could provide the reversible and subtle conformational change with the small difference of fluorescent and CD spectra. TPase is so flexible that it can form closed or open conformations according to variations of the coordination geometry of bound cation [20, 21]. Structurally-flexible TPase produced the appearance of TPase activity on D-serine through the subtle conformational change.

\subsection{Possibility of D-serine racemization}

There may be the possibility that D-serine exposed to $60{ }^{\circ} \mathrm{C}$ for $6 \mathrm{hr}$ in $1.2 \mathrm{M}$ DAP solution is racemized to L-serine. Thus, we studied carefully by circular dichroism spectrometry whether or not D-serine was chemically racemized into L-serine in this 
reaction condition. $20 \mathrm{mM}$ DL-serine was first resolved for control on a Crownpack CR (+) HPLC column (Figure 7(a)). D- and L-serine were eluted at retention times of 19 min and 23 min, respectively. D-serine appeared on the negative side, and L-serine on the positive side. $10 \mathrm{mM}$ D-serine that was exposed to $60{ }^{\circ} \mathrm{C}$ for $6 \mathrm{hr}$ in $1.2 \mathrm{M}$ DAP was subsequently resolved. If L-serine is produced by D-serine racemization, any peak should be found on the positive side at or near a retention time of L-serine designated by arrow. But no positive peak was seen there (Figure 7(b)). This result indicated that D-serine was not racemized at all. We confirmed that D-serine served as a substrate to synthesize L-tryptophan.

\subsection{Enzymatic Assay of pyruvate production from D-serine}

Tryptophanase can degrade L-serine into pyruvate through $\beta$-elimination [22]. L-serine, after combining with TPase, looses a hydroxyl group to yield an $\alpha$-aminoacylate intermediate via an external aldimine intermediate [23]. The intermediate can add $\mathrm{H}_{2} \mathrm{O}$ to produce pyruvate. If indole is present, TPase can add indole to produce L-tryptophan by $\beta$-replacement with competitive suppression of pyruvate production [24]. Indole is a strong inhibitor of $\beta$-elimination on pyruvate formation from L-serine. Although TPase strikingly has the broad substrate specificity in which pyridoxal 5'-phosphate plus metal ions catalyze pyruvate formation from L-serine, L-cysteine, and many other $\beta$-substituted amino acids, and also the synthesis of tryptophan from L-serine and indole, there is no possibility that D-serine serves as substrate in these degradation and synthesis reactions [25]. However, it is not the case when DAP exists. In Fig. 8, pyruvate production was calculated on the basis of the decrease of optical density at $340 \mathrm{~nm}$ responsive to the oxidation reaction from NADH 
to $\mathrm{NAD}^{+}$that was coupled to the reduction reaction from pyruvate to lactate by lactate dehydrogenase. When TPase reacted with D-serine at $60{ }^{\circ} \mathrm{C}$ for $8 \mathrm{hr}$ in the presence of 1.2 M DAP, pyruvate was increasingly produced with reaction time (Fig. 8(a)). Pyruvate production from D-serine was quantitatively near to L-tryptophan production in Fig. 3 and 4. TPase can also synthesize L-tryptophan from indole, pyruvate and ammonium [26], and so there may be the possibility that pyruvate from D-serine is reused for L-tryptophan synthesis. We investigated TPase activity on pyruvate production towards various concentrations of indole. Fig. 8(b) showed it decreased against increasing indole concentrations to halt perfectly over $5 \mathrm{mM}$ of indole. In Fig 3 and 4, L-tryptophan synthesis was analyzed at $5.4 \mathrm{mM}$ of indole, whose concentration fell into a condition of the perfect cessation of the pyruvate formation.

\subsection{Reaction pathway of L-tryptophan synthesis from D-serine}

Tryptophanase with absolute stereospecificity cannot react with D-serine in usual condition, but can react with D-serine in the presence of DAP. The racemization of D-serine to L-serine was first suspected as a possible cause of this activity. However, no racemization of D-serine was shown in Fig. 7. D-serine is incorporated directly into TPase active site as a substrate. D-serine binds at the active site of TPase to produce TPase-bound D-serine, which is converts to TPase-bound aminoacrylate. It is followed by two possible reaction pathways for L-tryptophan synthesis, in which pathway the TPase-bound aminoacrylate is the key common intermediate. One is $\beta$-elimination route and the other $\beta$-replacement. If the TPase-bound aminoacrylate takes the $\beta$-elimination reaction pathway, it produces pyruvate. The pyruvate product is utilized for L-tryptophan synthesis together with indole and ammonia. On the other hand, if the 
TPase-bound aminoacrylate intermediate follows the $\beta$-replacement reaction pathway, it produces L-tryptophan via a TPase-bound quinonoid intermediate. This $\beta$-replacement reaction is the same reaction pathway as tryptophan synthase, though no structural similarity is detected between the two enzymes [27, 28]. In Fig. 3 and 4, L-tryptophan synthesis was analyzed at an indole concentration of $5.4 \mathrm{mM}$ at which pyruvate production was stopped. This indicated that L-tryptophan was synthesized from D-serine without passage of $\beta$-elimination. However, we still suspected the involvement of pyruvate in L-Trp synthesis from D-Ser. We made an additional experiment to check the absence or presence of L-Trp synthesis in the presence of $0.7 \mathrm{mM}$ pyruvate, which was equal to the pyruvate production degraded from D-Ser by $\beta$-elimination for $6 \mathrm{~h}$ and at $60{ }^{\circ} \mathrm{C}$. L-Trp was analyzed with UV and CD detectors on a HPLC resolution column. As a result, L-Trp could not be detected at all (data unshown). This unambiguously shows that pyruvate is not involved in L-Trp synthesis in the present reaction system. Thus, we confirm that L-tryptophan synthesis from D-serine takes place via the $\beta$-replacement reaction pathway.

\subsection{Unexpectedly-low barrier excluding D-amino acid from L-amino acid}

Tryptophanase is so flexible that domain shifts can be observed with the holo form found in an open conformation and the apo form in open, wide-open, or closed conformation depending on the nature of different bound ions [29]. TPase produces the broad substrate specificity on many $\beta$-substituted L-amino acids owing to the conformationally-flexiblity. As far as the stereospecificity is concerned, it remains unchanged. Many enzymes are known to bind both enantiomeric forms of a substrate. When the catalytic reaction is restricted to one enantiomer, the other enantiomer, 
although bound to the active site, is inert or it functions as an inhibitor $[4,30]$. Generally speaking, it is impossible to transform a D-amino acid into an active substrate. Additionally, synthetic reactions will especially require more absolute stereospecificity than degradative reactions because the former is the reaction to create life, which makes it more difficult to find versatile stereospecificity in the synthesis reaction. However, inactive D-serine becomes active in the presence of DAP. As far as we know, there have been no previous reports in which an enzyme with tough stereospecificity bears activity to a D-amino acid. A very subtle conformational change, which is detected by fluorescence and circular dichroism spectrometry, transforms D-serine from an inactive to an active form. A barrier discriminating between D- and L-amino acid, which serves as the driving force for chiral homogeneity, only has very slight structurally-difference. Although it is reasonable to predict that L-dominant asymmetric selection system can only be built on a solid foundation of absolute enzyme stereospecificity, in fact quite the contrary happened. We should break away from the conventional idea that the enzyme stereospecificity has absolute immutability and stability. The enzyme stereospecificity is underpinned by a delicate balance between conformation and saline environment. Although we have yet to elucidate how the exclusive selection of L-amino acid, or the origin of homochirality occurred in primitive earth, this study suggests the saline environment is one significant factor to produce chiral homogeneity. Our results that show D-serine is catalyzed as a substrate in the synthesis reaction, providing a good clue to solve this difficult problem.

\section{Conclusion}


TPase produces pyruvate from D-serine via a $\beta$-elimination reaction pathway in the presence of DAP, but when indole is present, it can synthesize L-tryptophan via $\beta$-replacement without pyruvate production. Although D-serine is excluded from the original metabolism of TPase in ordinary reaction condition, D-serine can be accepted as an active substrate through a small stereostructual change which DAP provokes in TPase. Tryptophanase-catalyzed L-tryptophan synthesis system from D-serine supplies an attractive model to study the origin of homochirality.

\section{Acknowledgments}

The authors would like to thank the Project Research Program of the Kyoto University Research Reactor Institute for supporting partly our research.

\section{References}

[1] M. Lahav, Orig. Life Evol. Biosph. 37 (2007) 371.

[2] W. A. Bonner, Origins Life Evol. Biosph. 25 (1995) 175.

[3] G. Popjak, In: The Enzymes, volume, Academic Press, New York, 3rd ed., 1970, p.115.

[4] R. Bentley, Arch. biochem. Biophys. 414 (2003) 1.

[5] E.E. Snell, Adv. Enzymol. Relat. Areas Mol. Biol. 42 (1975) 287.

[6] A. Shimada, I. Nakamura, Viva Origino 20 (1992) 147.

[7] A. Shimada, H. Shishido, I. Nakamura, Amino Acids 11 (1996) 83.

[8] A. Shimada, H. Kogure, H. Shishido, I. Nakamura, Amino Acids 12 (1997) 379. 
[9] A. Shimada, H. Shishido, H. Kogure, I. Nakamura, N. Fuji, M. Akaboshi, In: M. Akaboshi (Eds), The Role of Radiation in the Origin and Evolution of Life, Kyoto University Press, Kyoto, 2000, p. 243.

[10] R. S. Phillips, Biochemistry 30 (1991) 5927.

[11] D.M.R. Mateus, S.S. Alves, M.M.R. Fonseca, J. Biosci. Bioeng. 5 (2004) 289.

[12] A. Shimada, N. Fujii, T. Saito, In: G. Paliyi, C. Zucchi, L. Caglioti (Eds.), Progress in Biological Chirality; Elsevier, Oxford, 2004, p. 321.

[13] A. Shimada, H. Ozaki, N. Fujii, T. Saito, Int. J. Mol. Sci. 10 (2009) 2578.

[14] A. Shimada, S. Yuasa, Viva Origino 8 (1979), 23.

[15] S. Yuasa, A. Shimada, Sci. Rep. Osaka Univ. 31 (1982) 13.

[16] S. Yuasa, M. Itoh, A. Shimada, J. Chromatogr. Sci. 22 (1984) 287.

[17] G. Musci, L.J. Berliner, Biochemistry 24 (1985) 3852.

[18] T. Kortemme, M. RamirezAlvarado, L. Serrano, Science, 281 (1998) 253.

[19] Y. Kuroda, Y. Maeda, S. Sawa, K. Shibata, K. Miyamoto, T. Nakagawa, J. Pept. Sci. 9 (2003) 212.

[20] S. Ku, P. Yip, P.L. Howell, Acta Crystallogr. D Biol. Crystallogr. D62 (2006) 814.

[21] A. Kogan, G.Y. Gdalevsky, R. Cohen-Luria, Y. Goldgur, R.S. Phillips, A.H. Parola, O. Almog, BMC Struct. Biol. 9 (2009) 65.

[22] L.N. Zakomirdina, V.V. Kulikova, O.I. Gogoleva, I.S. Dementieva, N.G. Faleev, T.V. Demidkina, Biochemistry (Moscow) 67 (2002) 1189-1196.

[23] V.V. Kulikova, L.N. Zakomirdina, N.P. Bazhulina, I.S. Dementieva, N.G. Faleev, P.D. Gollnick, T.V. Demidkina, Biochemistry (Moscow) 68 (2003) 1181-1188.

[24] Y. Morino, E.E. Snell, J. Biol. Chem. 242 (1967) 2793.

[25] W.A. Newton, Y. Morino, E.E. Snell, J. Biol. Chem. 240 (1965) 1211.

[26] T. Watanabe, E.E. Snell, Proc. Nat. Acad. Sci. 69 (1972) 1086. 
[27] M.N. Isupov, A.A. Antson, E.J. Dodson, G.G. Dodson, I.S. Dementieva, L.N.

Zakomirdina, K.S. Wilson, Z. Dauter, A.A. Lebedev, E.H. Harutyunyan, J. Mol. Biol. $276(1998) 603$.

[28] E.W. Miles, The Chemical Record, 1 (2001) 140.

[29] N. Tsesin, A. Kogan, G.Y. Gdalevsky, J. Himanen, R. Cohen-Luria, A.H. Parola, Y. Goldgur, O. Almog, Acta Crystallogr. D Biol .Crystallogr. D63 (2007) 969.

[30] S. K. Griffiths, R. D. DeMoss, J. Bacteriol. 101 (1970) 810. 


\section{Figure captions}

Fig. 1. Cellulose thin layer chromatography of the tryptophan synthesized from Lor D-serine by TPase. For reference, D- and L-tryptophan were developed in the middle. lane1: L-serine + indole + pyridoxal 5'-phosphate + TPase without DAP, lane 2: D-serine + indole + pyridoxal 5'-phosphate $+1.2 \mathrm{M}$ DAP without TPase, lane 3: D-serine + indole + pyridoxal 5'-phosphate + TPase without DAP, lane 4: D-serine + indole + pyridoxal 5'-phosphate + TPase + 1.2 M DAP.

Fig. 2. Resolution chromatograms of reaction product. (a and b) Advanced resolution chromatography of DL-tryptophan detected with (a) UV and (b) CD monitor. (c and d) L-tryptophan synthesized from L-serine was detected with (c) UV and (d) CD monitor. (e) No peak near retention time of 30 min in the absence of DAP. (f and g) L-tryptophan synthesized from D-serine was detected with (f) UV and (g) CD monitor in the presence of DAP.

Fig. 3. Tryptophanase-catalyzed L-tryptophan synthesis from L- or D-serine varying with DAP concentrations at a reaction temperature of $60^{\circ} \mathrm{C}$.

Fig. 4. L-tryptophan synthesis from L-serine or D-serine with reaction temperature at a fixed 0 or $1.2 \mathrm{M}$ concentration of DAP.

Fig. 5. Relative fluorescence intensity of bis-ANS-bound TPase in PB, DAP and MAP solutions. 
Fig.6. Circular dichroism spectra of TPase in PB, DAP and MAP solutions.

Fig. 7. Resolution chromatograms of D-serine. (a) DL-serine resolution for control; (b) D-serine exposed to $60{ }^{\circ} \mathrm{C}$ for $6 \mathrm{hr}$ in $1.2 \mathrm{M}$ DAP solution.

Fig. 8. Enzymological assay of pyruvate produced from D-serine in the presence of DAP. Reaction mixtures including $0.4 \mathrm{mM}$ pyridoxal 5'-phosphate, $20 \mathrm{mM}$ D-serine, $0.46 \mu \mathrm{M}$ TPase and 1.2 M DAP reacted at $60{ }^{\circ} \mathrm{C}$ for 0 to $8 \mathrm{hr}$ in the absence of indole

((a) Pyruvate production with reaction time), or at $60{ }^{\circ} \mathrm{C}$ for $6 \mathrm{hr}$ with 0 to $8 \mathrm{mM}$ indole ((b) Decreasing pyruvate formation against increasing indole). 
Fig. 1

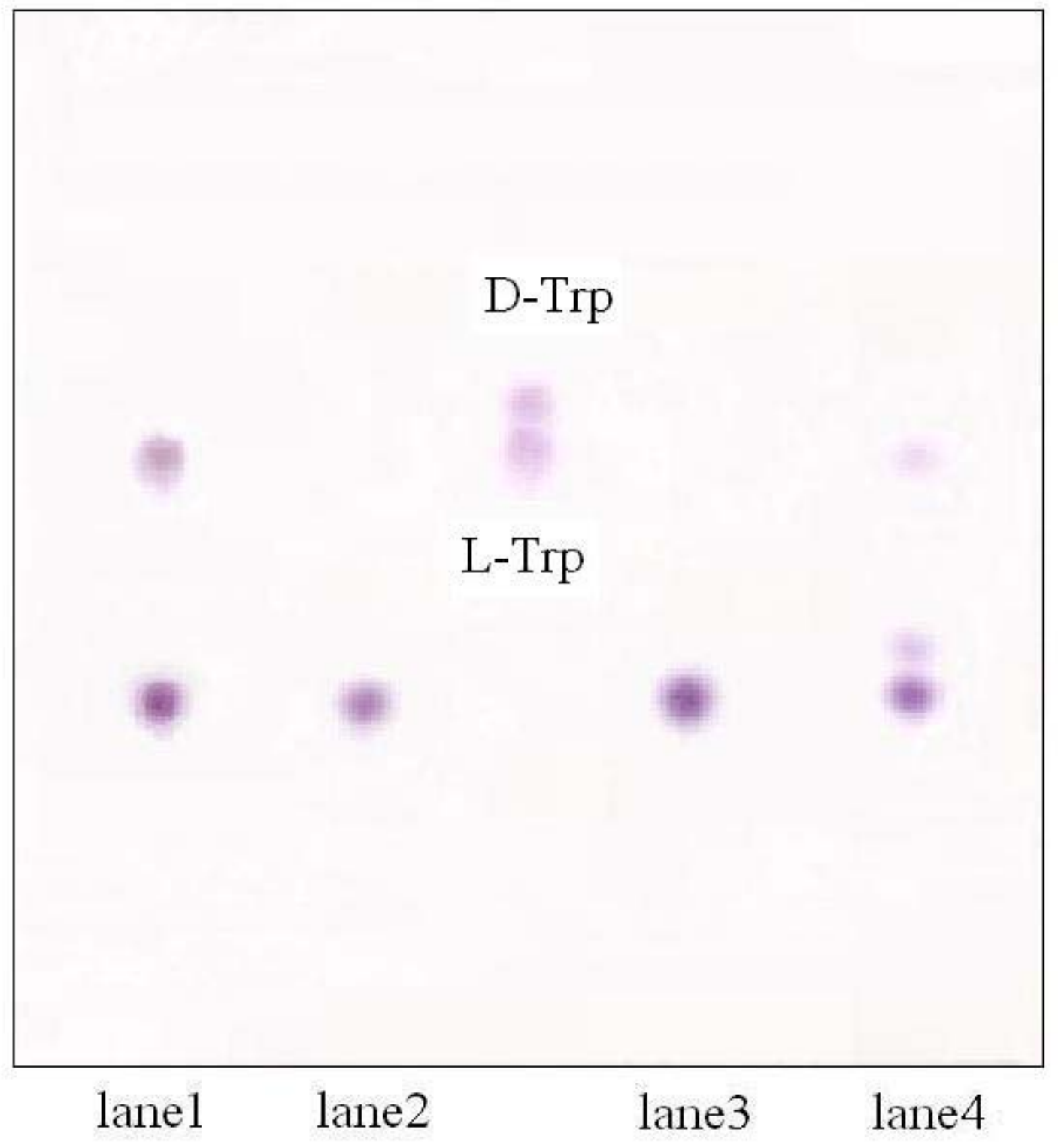


Fig. 2(a) (g)
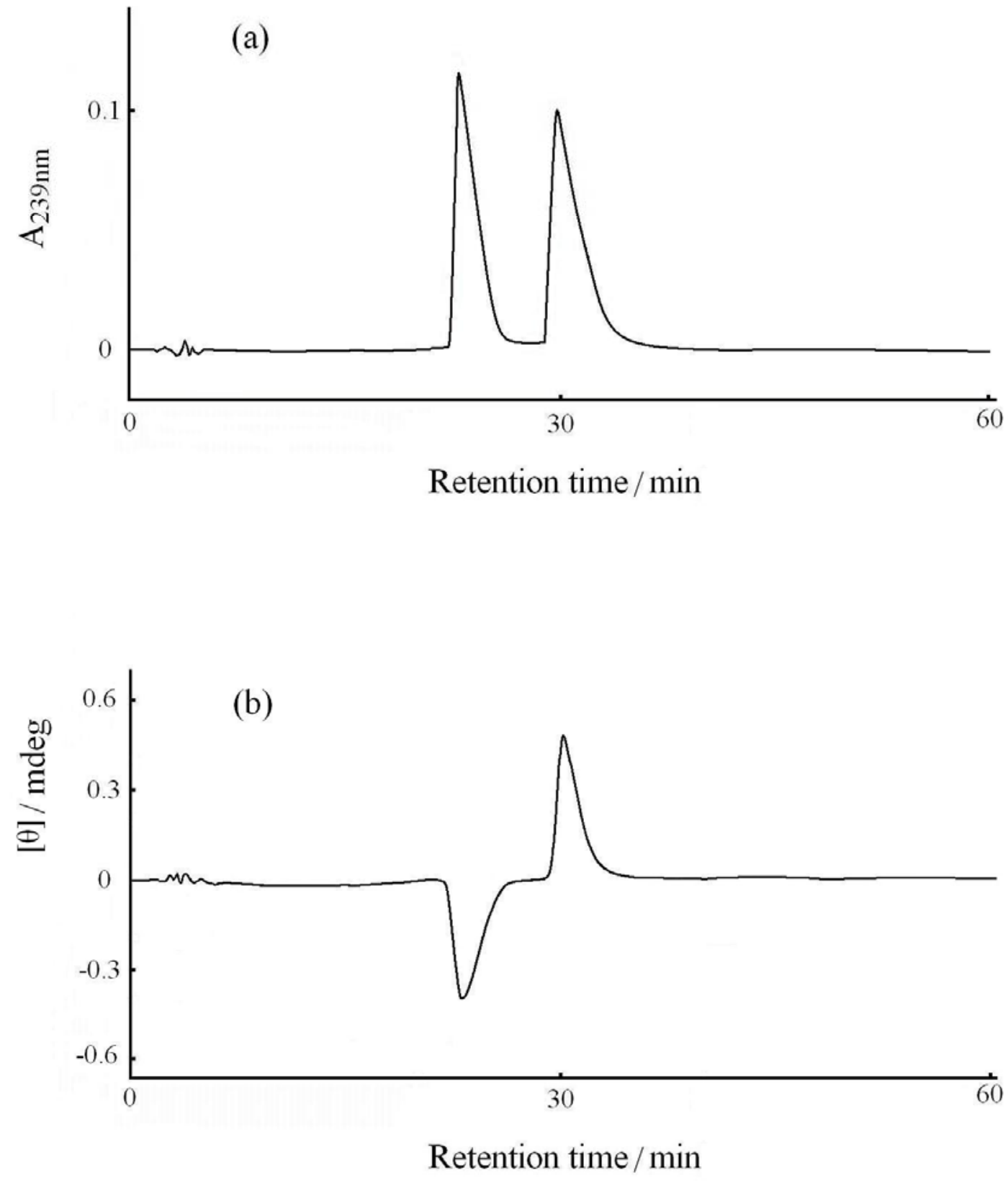

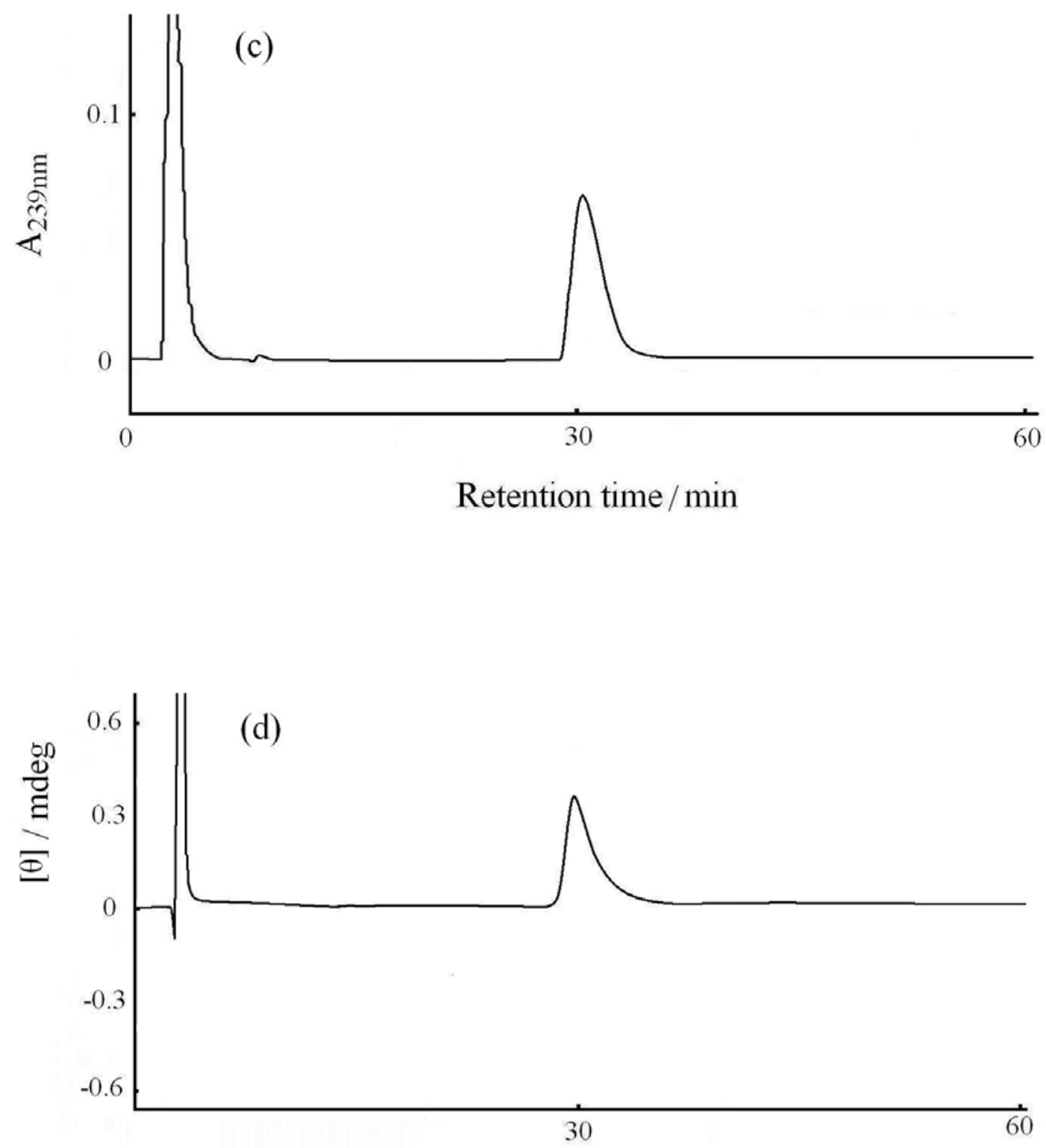

Retention time / min 

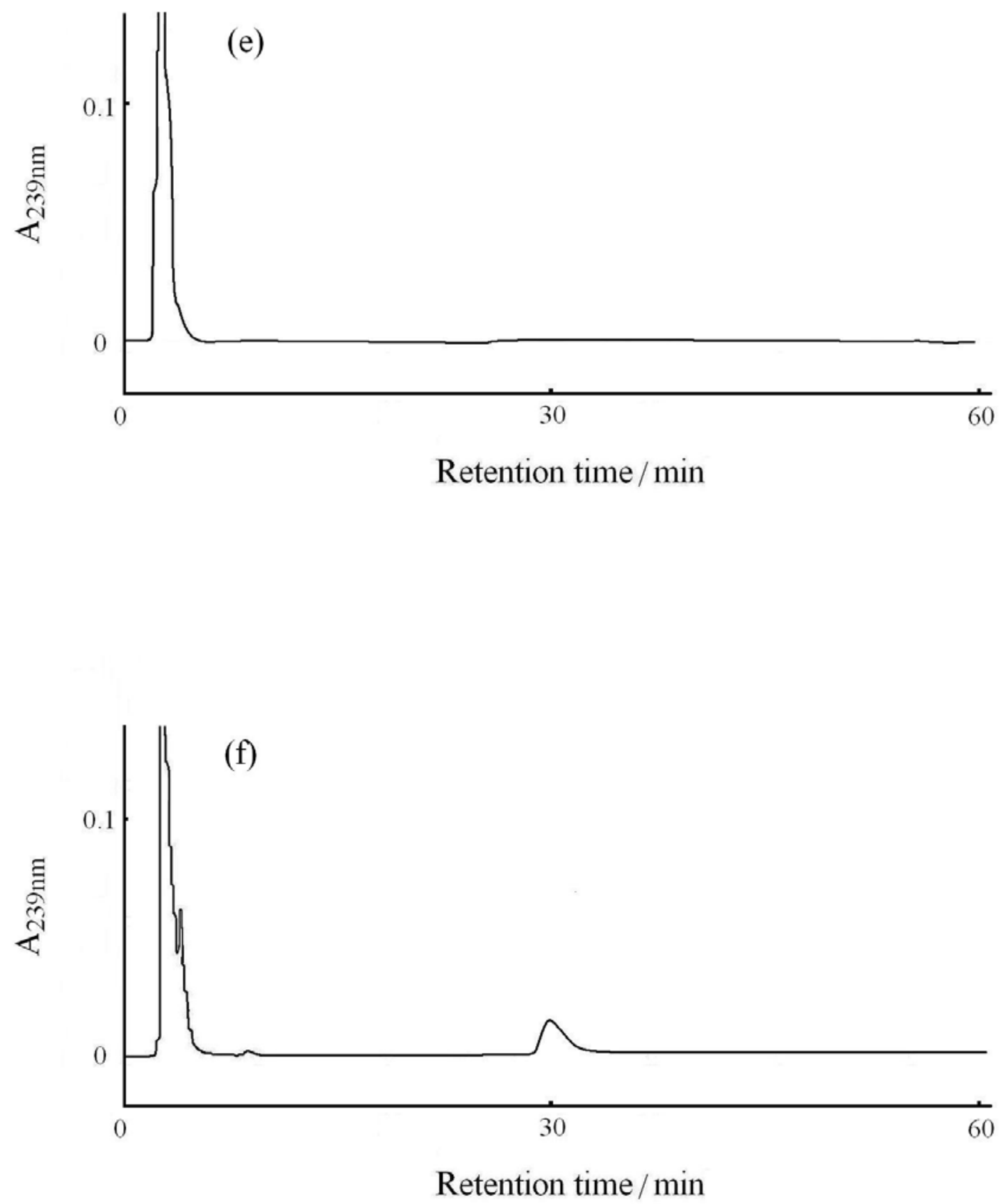


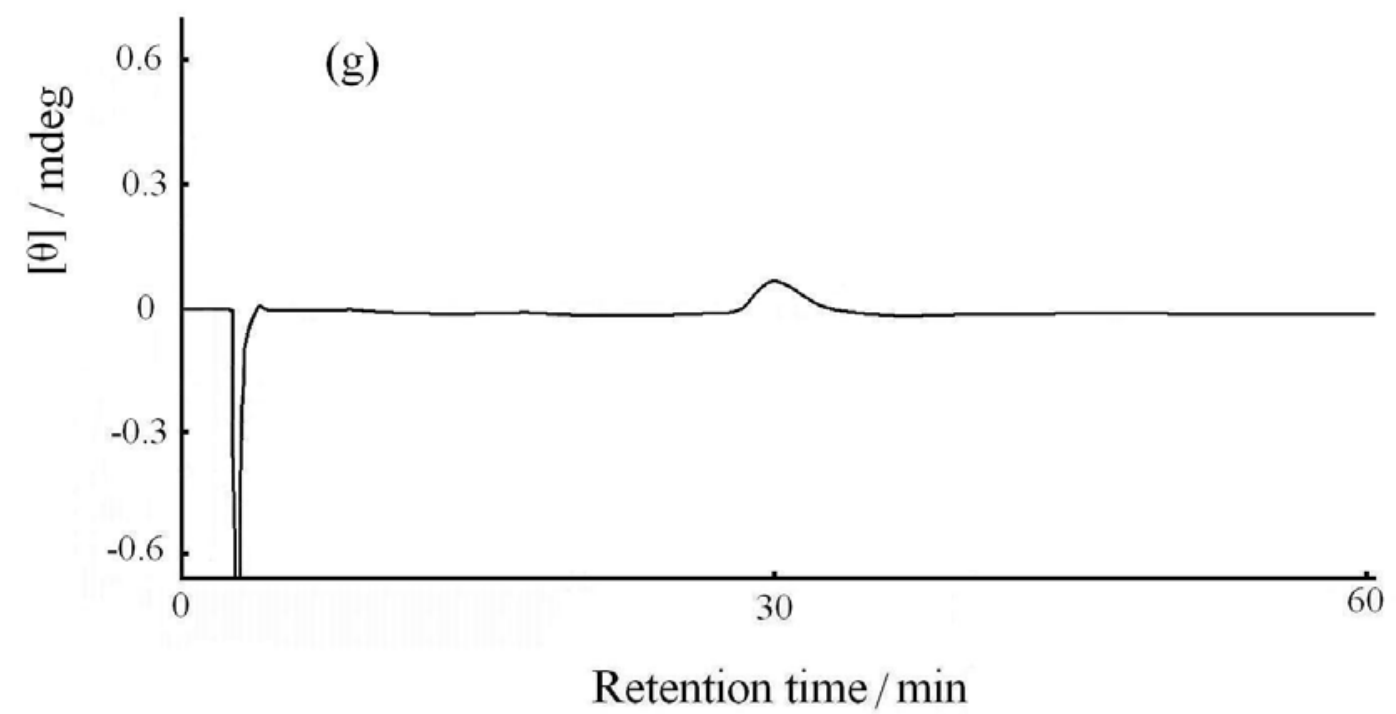


Fig. 3

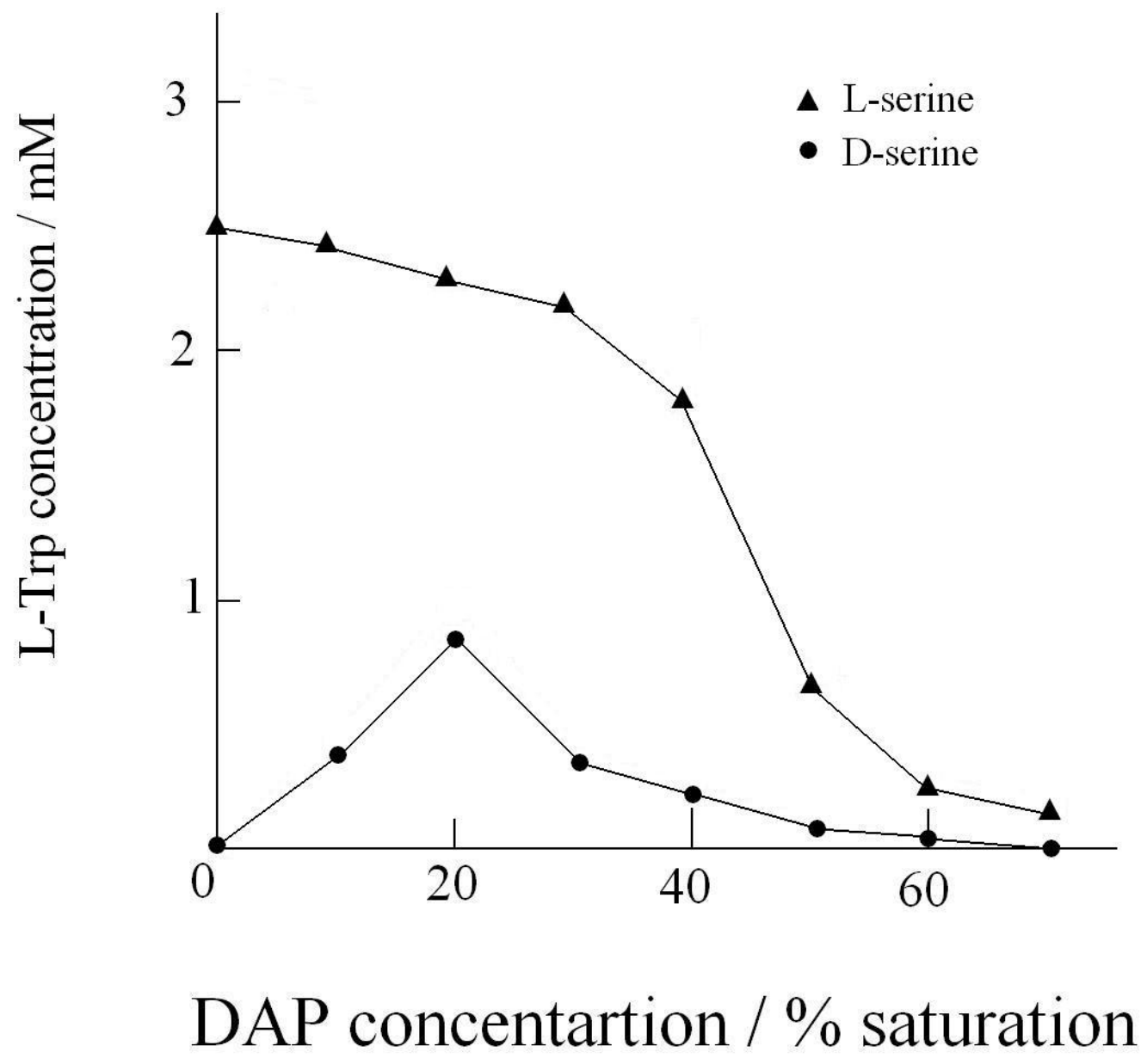


Fig. 4

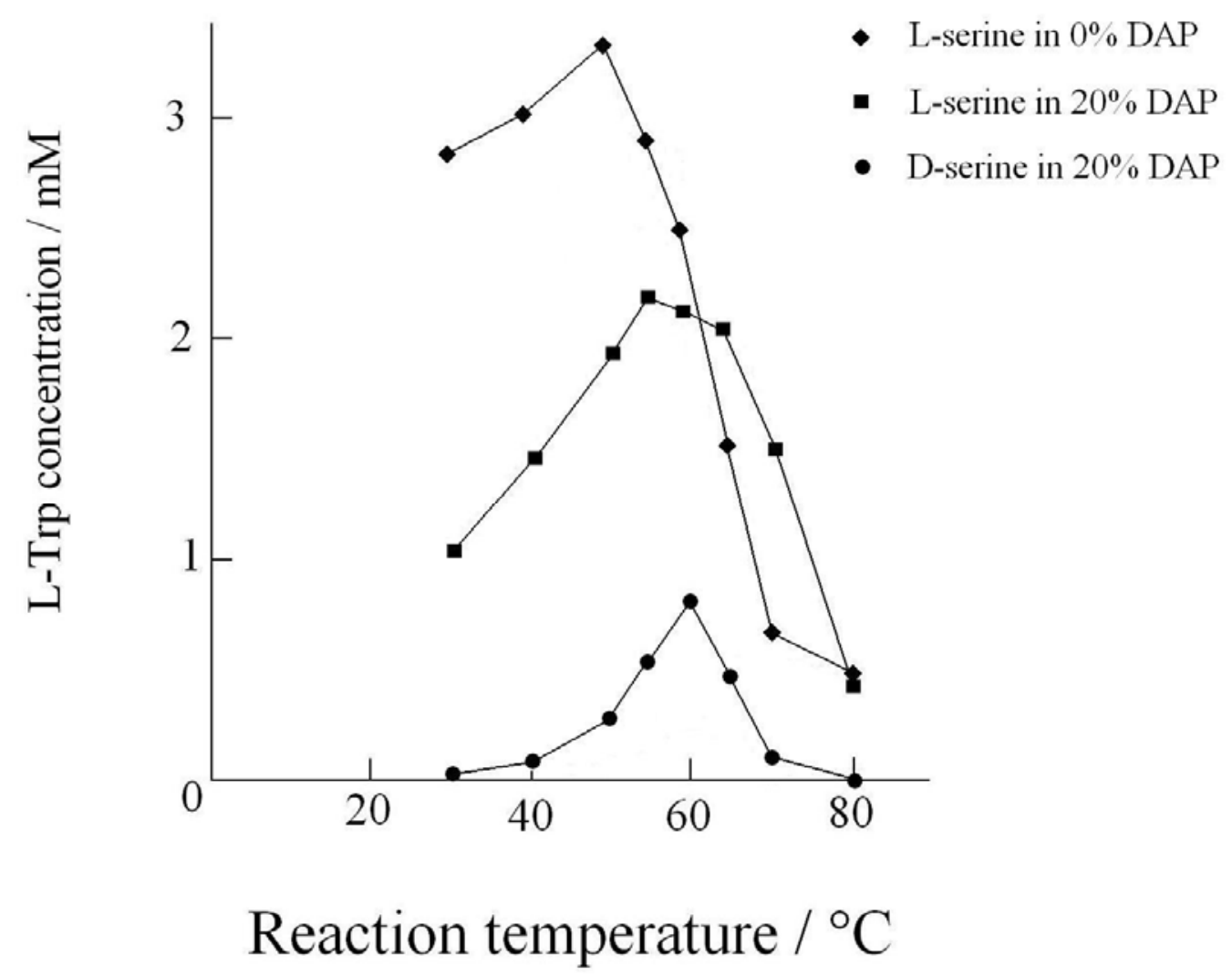


Fig. 5

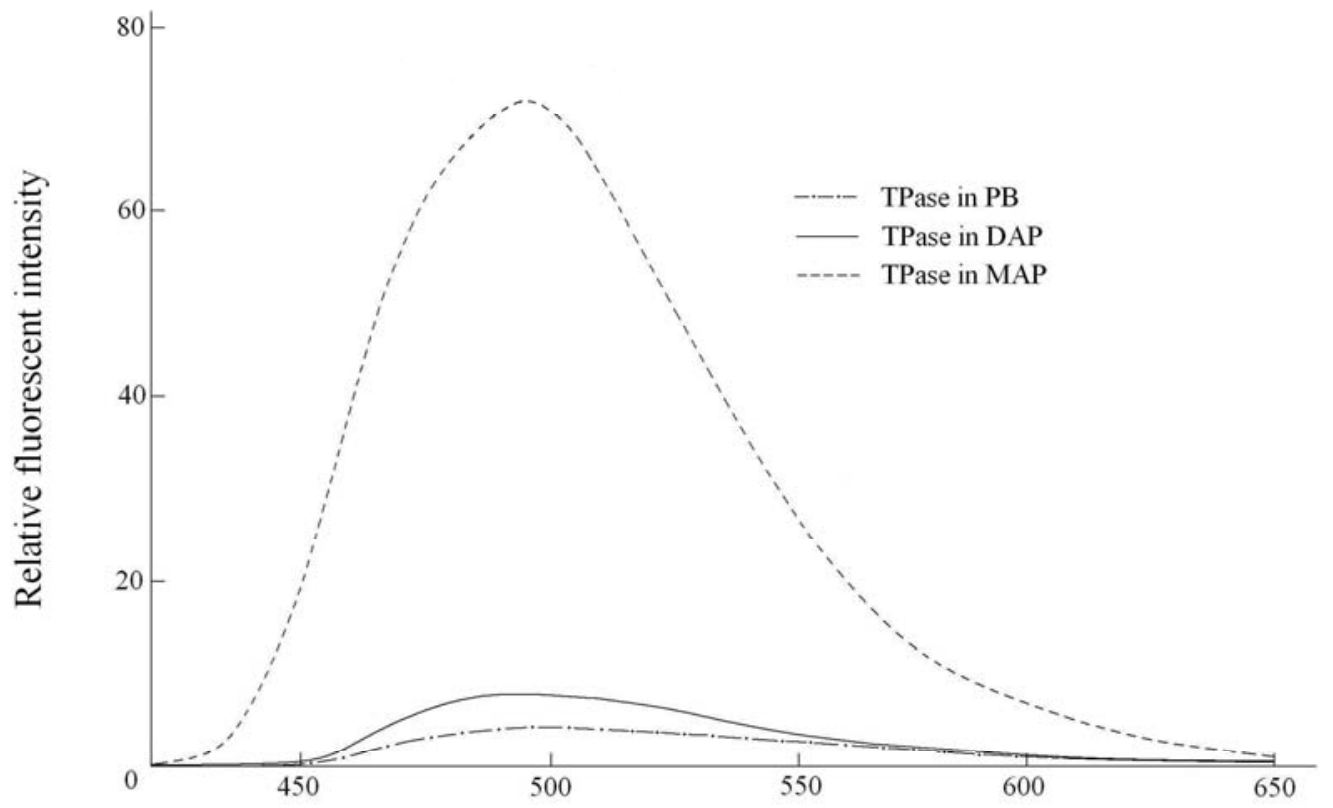

Wavelength / nm 
Fig. 6

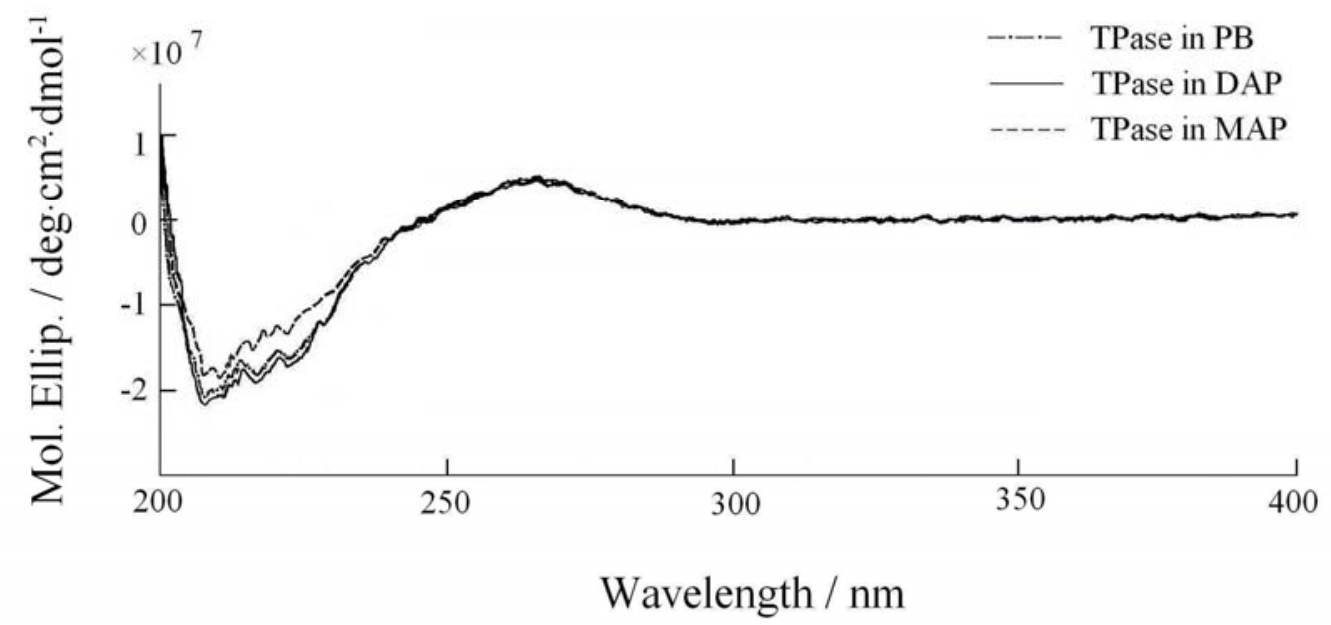


Fig. 7(a)

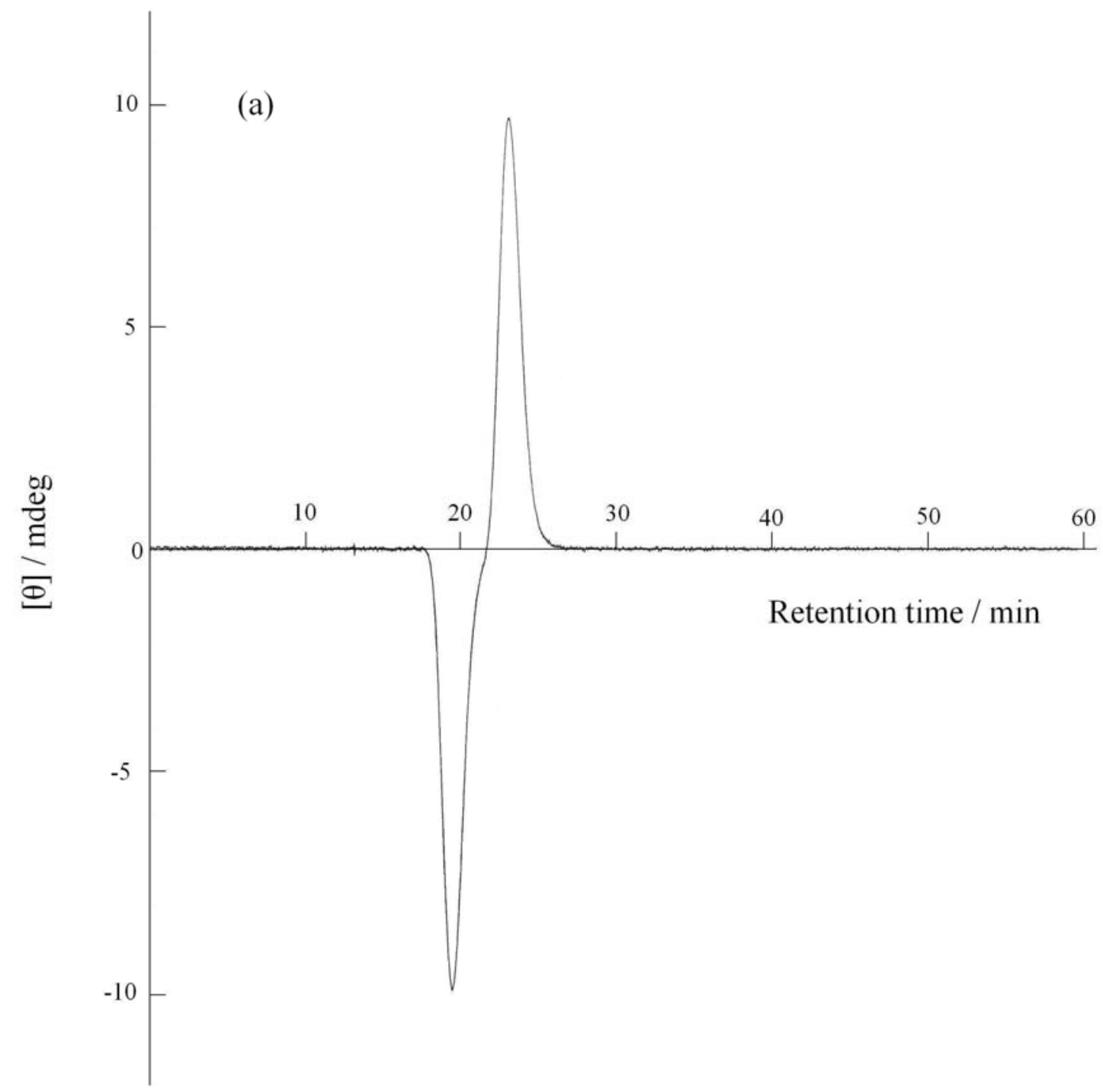


Fig.7(b)

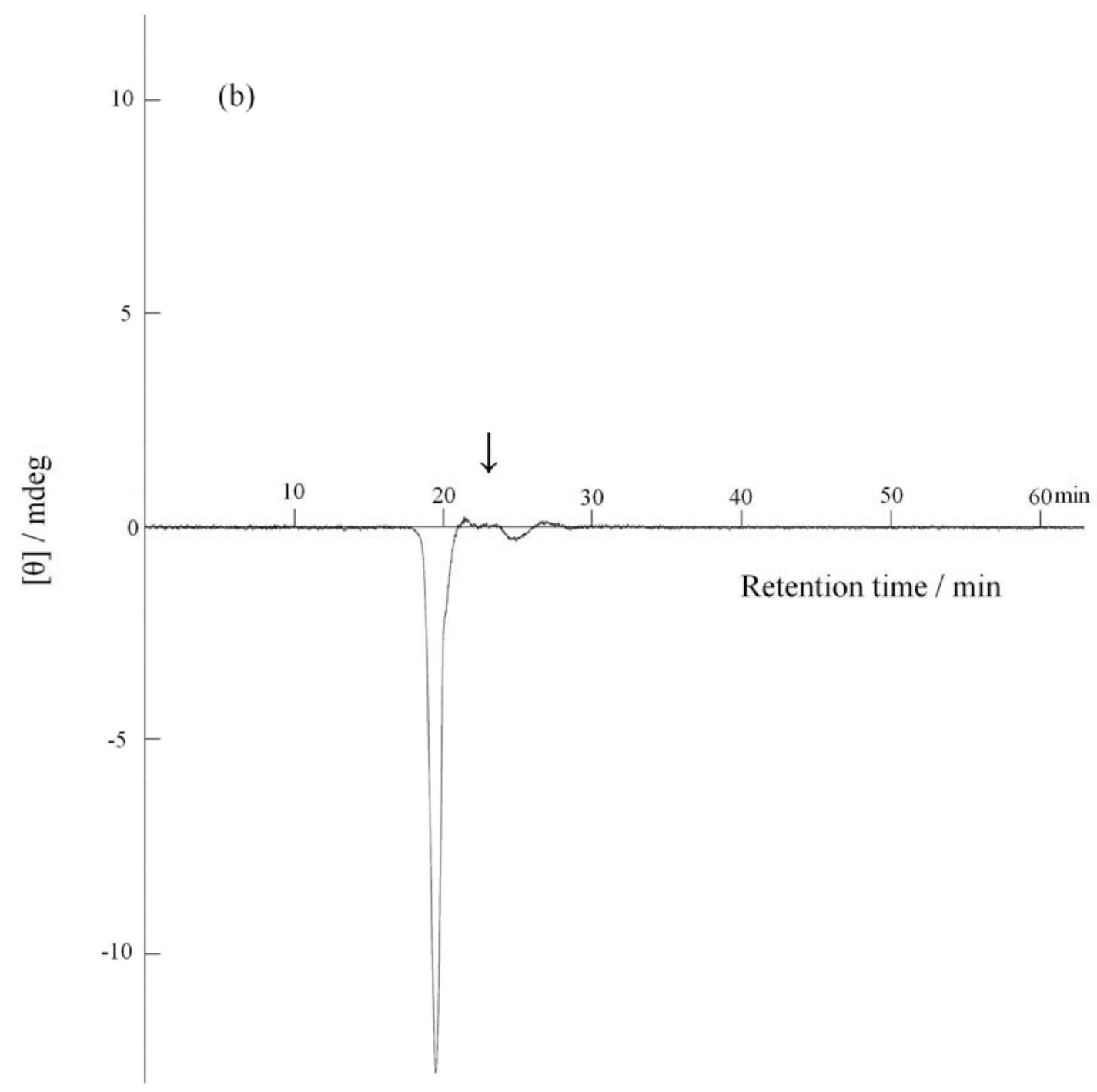


Fig. 8 (a)

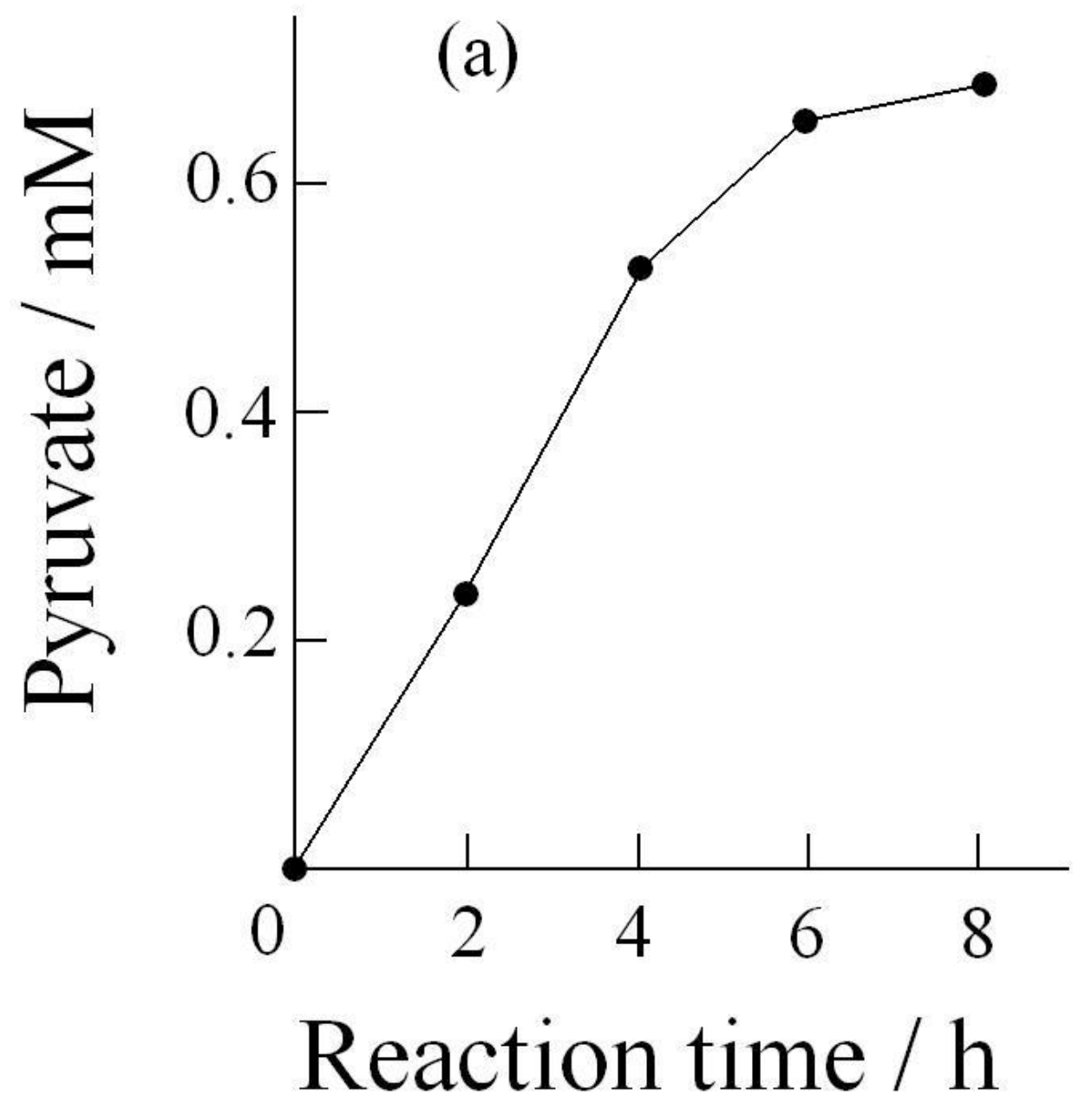


Fig. 8(b)

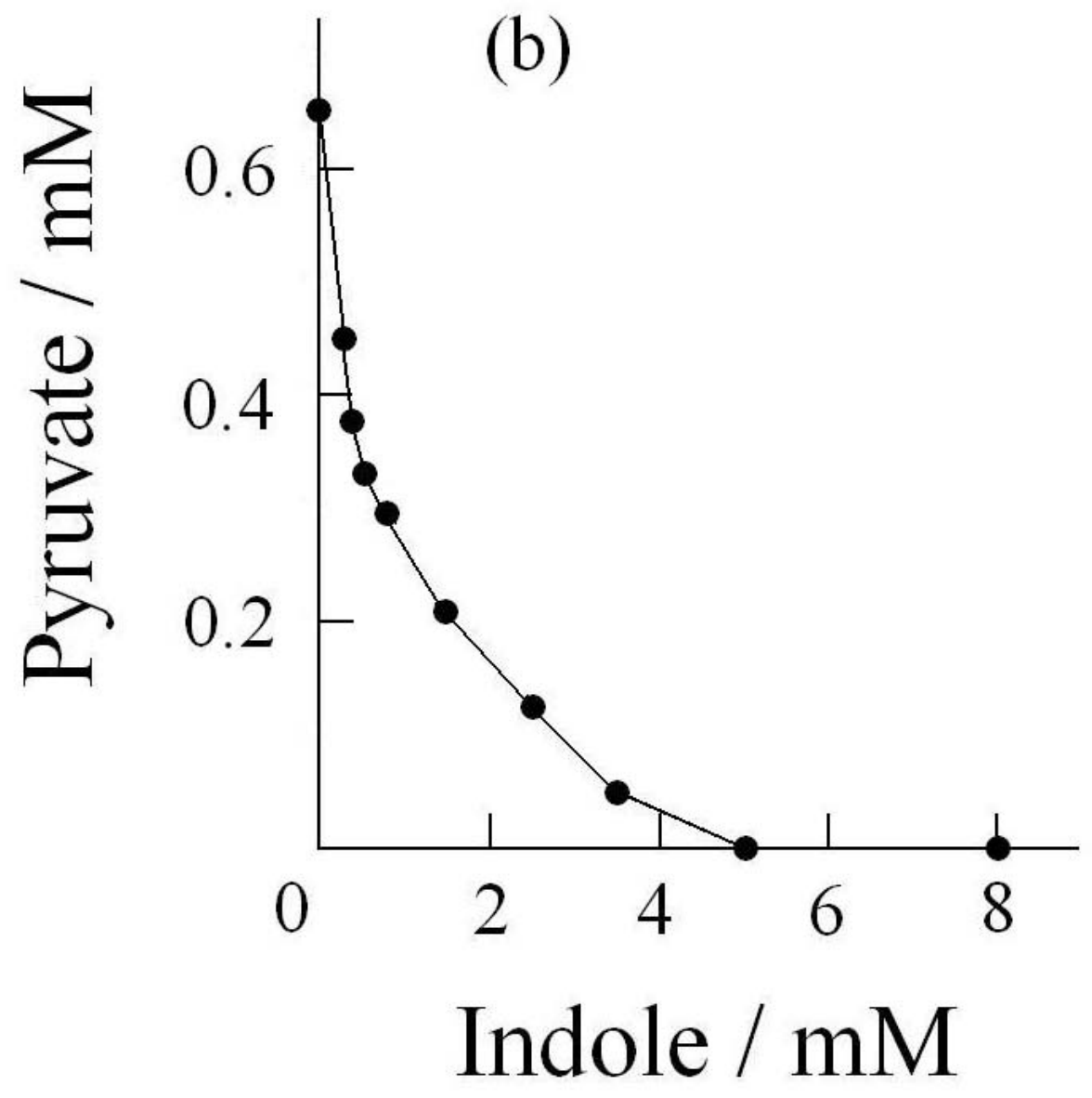

